Quim. Nova, Vol. 34, No. 9, 1562-1568, 2011

\title{
NITRATO DE AMÔNIO OCLUÍDO EM ZEÓLITA 4A: LIXIVIAÇÃO E ABSORÇÃO DE NITROGÊNIO NO CULTIVO DE MILHO
}

\author{
Jardel Cavalcante Rolim de Almeida Andrade e Lindomar Roberto Damasceno da Silva* \\ Departamento de Química Orgânica e Inorgânica, Universidade Federal do Ceará, CP 6031, Campus Universitário do Pici, \\ 60451-970 Fortaleza - CE, Brasil \\ Ismail Soares e Ricardo Espíndola Romero \\ Departamento de Ciências do Solo, Universidade Federal do Ceará, Av. Mister Hull, 12200, Campus Universitário do Pici, \\ 60451-970 Fortaleza - CE, Brasil
}

Recebido em 9/1/11; aceito em 4/5/11; publicado na web em 4/7/11

\begin{abstract}
NITRATE OCCLUDED IN ZEOLITE 4A: ABSORPTION AND LEACHING OF NITROGEN IN THE CULTIVATION OF CORN. Systematic studies were undertaken in the intra zeolitic media to better understand the ability of zeolite type LTA in occluded nitrogen used in fertilizer and soil conditioning. We have measured the dry matter production from the cultivation of corn in a greenhouse for about 40 days, and also the amounts of nitrogen absorbed, retained and lost by leaching. The dry matter production in the cultivation with different concentrations of nitrogen occluded in the zeolite, was more efficient than the traditional fertilizer, which demonstrated better use of nitrogen to reduce leaching losses, which implies a possible reduction of costs for nitrogen.
\end{abstract}

Keywords: occlusion; release of nitrogen; fertilization.

\section{INTRODUÇÃO}

Com a tendência de crescimento do mercado agrícola, pesquisadores têm apresentado novos sistemas de cultivo, como os protegidos (túneis e estufas) e o hidropônico, em alternativa ao sistema tradicional do campo. Além destas, existe uma possibilidade de cultivo pouco empregada que é o zeopônico, no qual plantas são cultivadas em substrato artificial composto pelo aluminossilicato zeólita, ${ }^{1}$ que funciona como um sistema de liberação controlada e renovável de nutrientes minerais para as plantas. ${ }^{2}$ Este mineral apresenta três propriedades principais que conferem grande interesse para uso na agricultura: alta capacidade de troca de cátions, alta capacidade de retenção de água livre nos canais e alta habilidade na adsorção. ${ }^{3}$ Assim, a zeólita pode atuar na melhoria da eficiência do uso de nutrientes quanto à sua disponibilidade, na melhora do aproveitamento do nitrogênio $\left(\mathrm{NH}_{4}^{+}\right.$e $\left.\mathrm{NO}_{3}^{-}\right)$e redução das perdas por lixiviação dos cátions trocáveis, especialmente $\mathrm{K}^{+}$, e também como um fertilizante de liberação lenta. ${ }^{4,5}$

Quando as zeólitas são tratadas com sais no estado sólido ou fundido, ocorrem substituições das água de adsorção, dentro dos poros da zeólita. ${ }^{6}$ Dependendo do tamanho da janela dos poros da zeólita, o sal introduzido pode ser estabilizado através de ajuste geométrico e/ou interação eletrostática com a zeólita.

Os pesquisadores relatam que, além do nitrato de amônio, diversos outros sais poderiam ser ocluídos em estruturas zeolíticas e argilas. ${ }^{7-10}$

Zeólitas ocluídas com sais possuem dois tipos diferentes de fontes de nutrientes para plantas, cátions trocáveis de sua própria estrutura e o sal ocluído. Suas estruturas acomodam muito mais nutrientes no estado sólido do que através de troca catiônica em solução aquosa. Com isso, estudos sistemáticos sobre as propriedades de sais ocluídos têm se mostrado necessários para melhor compreensão dos mecanismos que permitiriam que as zeólitas pudessem ser utilizadas na estabilização de fertilizantes e como condicionadores de solos. ${ }^{2,11,12}$

\footnotetext{
*e-mail: lindomar@dqoi.ufc.br
}

Para a região tropical, há uma necessidade de se desenvolver tecnologias próprias para obtenção de matérias-primas e novos fertilizantes voltados às condições geoclimáticas para os solos desta região. Neste contexto, os minerais industriais, ressaltando as zeólitas, têm uma importância significativa na agricultura, podendo ser aplicados como condicionador de solos ou carreador de nutrientes.

Dessa forma, sabendo-se que a qualidade final de um fertilizante é resultante, dentre outros fatores, dos níveis de fornecimento de nutrientes ${ }^{13,14}$ e que na interação entre $\mathrm{NH}_{4} \mathrm{NO}_{3}$ e a zeólita ocorre o emparelhamento do nitrato com o íon amônio, que interage com o sistema poroso da zeólita, ${ }^{15}$ o presente trabalho objetivou avaliar o comportamento do nitrogênio ocluído nos poros da zeólita 4A, bem como os aspectos quantitativos, tais como, absorção, retenção e perda por lixiviação desta espécie a partir do comportamento térmico, buscando uma forma de cultivo de milho menos dispendiosa, com acompanhamento baseado na produção de matéria seca a partir de ensaios em casa de vegetação.

\section{PARTE EXPERIMENTAL}

\section{Oclusão de $\mathrm{NH}_{4} \mathrm{NO}_{3}$ em poros da zeólita $4 \mathrm{~A}$}

No estudo da oclusão de nitrogênio a partir do $\mathrm{NH}_{4} \mathrm{NO}_{3}$, foi utilizada uma zeólita do tipo LTA. ${ }^{16} \mathrm{O}$ procedimento foi realizado conforme descrito por Park e colaboradores ${ }^{2}$ com modificações, e consistiu em misturar zeólita 4A (sintetizada em escala piloto) e $\mathrm{NH}_{4} \mathrm{NO}_{3}$ através de interações físicas em moinho planetário (Fritsch modelo Planetary Mono "pulveresette 6") a 370 rpm durante 10 min, contendo 6 esferas com diâmetro de $1 \mathrm{~cm}$ em panelas de aço carbono 1400. A mistura foi posta em cadinho de teflon e aquecida em estufa a $185^{\circ} \mathrm{C}$ durante $8 \mathrm{~h}$.

Foram realizados estudos para se obter a melhor proporção $\mathrm{NH}_{4} \mathrm{NO}_{3} / \mathrm{NaA}$ conforme as seguintes porcentagens de $\mathrm{NH}_{4} \mathrm{NO}_{3} \mathrm{em}$ relação à massa de zeólita $\mathrm{NaA}: 20,40,60,80$ e $100 \%$. 
O fertilizante (zeólita + nitrogênio) para o ensaio agronômico em escala piloto foi obtido usando-se $70 \mathrm{~g}$ de zeólita e $42 \mathrm{~g}$ de $\mathrm{NH}_{4} \mathrm{NO}_{3}$, resultando em $60 \% \mathrm{~m} / \mathrm{m}$ de nitrogênio na zeólita $\left(60 \mathrm{~N} \_4 \mathrm{~A}\right)$. Ao final de cada etapa de oclusão, foram retiradas pequenas quantidades para análise de infravermelho (FT-IR) e termogravimetria (TG/DTG).

\section{Análises termogravimétricas}

Através de análises termogravimétricas (TG e DTG) foram realizadas investigações sobre a estabilidade termocinética do nitrato de amônio ocluído. ${ }^{17}$

Os estudos cinéticos de termodegradação foram realizados submetendo as amostras 40N_4A e 60N_4A, por apresentarem melhores rendimentos de oclusão, a razões de aquecimento de $2,5,10,15$ e $20{ }^{\circ} \mathrm{C} \mathrm{min}^{-1}$. O cálculo das energias de ativação para os processos termodegradativos foi realizado a partir do método de Ozawa, mediante a construção de um gráfico do logaritmo das razões de aquecimento versus o inverso da temperatura absoluta para os valores inerentes a cada fração de massa residual das análises termogravimétricas. Os parâmetros cinéticos foram obtidos através da equação $\mathrm{E}_{\mathrm{a}}=-(\mathrm{R} / \mathrm{b}) \cdot \Delta \log \beta / \Delta(1 / \mathrm{T})$, derivada das considerações de Ozawa, onde $\mathrm{E}_{\mathrm{a}}=$ energia de ativação (Arrhenius) estimada, $\mathrm{J} / \mathrm{mol} ; \mathrm{R}=$ constante dos gases, 8,314 $\mathrm{J} \mathrm{mol}^{-1} \mathrm{~K}^{-1} ; \mathrm{b}=0,457 / \mathrm{K} ; \mathrm{b}=$ razão de aquecimento, $\mathrm{K} / \mathrm{min}$; $\mathrm{T}=$ temperatura, $\mathrm{K}$.

\section{Coleta e caracterização do solo}

Para o cultivo do milho, foi coletado material de uma camada de 0-20 cm de profundidade de um Latossolo Vermelho Distrófico de textura argilosa, na fazenda Cercado do Meio - Quixeré (CE), $\left(5^{\circ} 06^{`} \mathrm{~S}\right.$ e $37^{\circ} 57^{\circ} \mathrm{O}$ ), numa área de clima semiárido (Chapada do Apodi). O solo foi seco em casa de vegetação a uma temperatura média de $30^{\circ} \mathrm{C}$, por um período de 30 dias e peneirado utilizando peneira de $2 \mathrm{~mm}$.

Foram realizadas análises de termogravimetria, difração de raios$\mathrm{X}$ e fluorescência de raios-X de uma amostra de solo in natura, bem como determinações físicas e químicas.

Para determinação do $\mathrm{pH}$ e condutividade do solo foram utilizadas suspensões aquosas em triplicata de aproximadamente $70 \mathrm{~g}$ de solo, cloreto de potássio $1 \mathrm{~mol} / \mathrm{L}$ e cloreto de cálcio $0,01 \mathrm{~mol} / \mathrm{L}$. Para determinar a porosidade do solo e estimar a quantidade de água a ser adicionada nos vasos, obteve-se a densidade do solo, pelo método da proveta, e a densidade das partículas, pelo método do balão volumétrico, para uma amostra deformada (solo solto, coletado com pá ou trado). ${ }^{18}$

Realizou-se também análise granulométrica (método da pipeta), que se baseia na velocidade de queda das partículas com dispersão física e química. Após as dispersões a suspensão foi submetida a um processo de separação nas frações areia, silte e argila.

\section{Preparo e condução do experimento}

O cultivo foi realizado em Fortaleza - CE ( $\left.3^{\circ} 44^{`} \mathrm{~S} ; 38^{\circ} 34^{\prime} \mathrm{O}\right)$, em casa de vegetação. A cultura de milho foi implantada em abril de 2009, em vaso de polietileno com capacidade para $3 \mathrm{~kg}, 2,5 \mathrm{~kg}$ de solo, sendo que os resultados foram obtidos entre maio e junho de 2009. O delineamento experimental utilizado foi o de blocos inteiramente casualizados, com 4 repetições por tratamento.

A análise de variância foi realizada pelo programa Sisvar, versão 5.1 (Departamento de Ciências Exatas da Universidade Federal de Lavras - DEX/UFLA; 1999-2007) e baseou-se nas médias das eficiências do aproveitamento de nitrogênio e produção de matéria seca, submetidas ao teste Tukey, a 5\% de probabilidade.

A solução nutritiva foi adicionada em cada vaso de acordo com as proporções dos macro e micronutrientes: $\mathrm{K}=1014,0 \mathrm{mg} ; \mathrm{Ca}=$ 600,0 mg; P = 186,0 mg; $\mathrm{Mg}=72,0 \mathrm{mg} ; \mathrm{S}=96,7 \mathrm{mg} ; \mathrm{Mn}=1,2 \mathrm{mg}$; $\mathrm{Cu}=0,05 \mathrm{mg} ; \mathrm{B}=1,0 \mathrm{mg} ; \mathrm{Mo}=0,2 \mathrm{mg} ; \mathrm{Fe}=0,006 \mathrm{mg}$ e $\mathrm{Zn}=1,7$ mg. Os fertilizantes nitrogenados livre $\left(\mathrm{NH}_{4} \mathrm{NO}_{3}\right)$ e ocluído na zeólita (60N_4A) foram adicionados nas proporções de 300, 500 e $700 \mathrm{mg}$ de $\mathrm{N}$ na forma de $\mathrm{NH}_{4} \mathrm{NO}_{3}$, e avaliados quanto a produção de matéria seca, tomando como base a testemunha (vaso sem nitrogênio). ${ }^{19}$

Foram semeadas com 6 sementes de milho (Embrapa BR 5011 sertanejo) a aproximadamente $3 \mathrm{~cm}$ de profundidade. $\mathrm{O}$ experimento foi conduzido por 38 dias, a contar da data de germinação, com adições de água desionizada a fim de que os vasos permanecessem em capacidade de campo, porém, coletando-se o excesso de água (drenada) para avaliar as perdas por lixiviação. Foram realizados 3 desbastes, o primeiro com 7 dias de germinação, descartando o material para permanecer 4 plantas por vaso; o segundo com 15 dias (permanecendo 2 plantas por vaso) e o terceiro (último) com 38 dias. As plantas do $2^{\circ}$ e $3^{\circ}$ desbaste foram colocadas em estufa a $60^{\circ} \mathrm{C}$ com circulação de ar durante uma semana. Quando secas, foram colocadas em dessecador para resfriar e, então, pesadas. Os parâmetros produção relativa (PR) e incremento de produção relativa (IPR) da matéria seca do milho foram obtidos para o solo pelas fórmulas: $\mathrm{PR}=($ Produção de MS do tratamento sem N x 100)/(Produção de MS do tratamento $\operatorname{com} \mathrm{N}$ ); IPR $=100-\mathrm{PR}$.

As amostras da parte aérea de cada vaso e a solução de lixiviação dos tratamentos tiveram as concentrações de nitrogênio determinadas por microdestilação de Kjeldahl em um analisador da marca TECNAL modelo TE-0363. ${ }^{20,21}$

\section{RESULTADOS E DISCUSSÃO}

Os espectros na região do infravermelho do $\mathrm{NH}_{4} \mathrm{NO}_{3} 98 \%$, da zeólita 4A e das etapas de oclusão do mesmo em poros da zeólita $\mathrm{NaA}$ são apresentados na Figura 1. Embora as condições físicas para incorporar o nitrato de amônio tenham sido severas, os espectros observados na Figura 1 indicam que o processo de oclusão não resulta em mudanças estruturais. Tal comportamento também tem sido citado por Yamada e colaboradores,${ }^{22}$ para experimentos realizados em condições similares. Tomando como referência a intensidade fixa da banda em $997 \mathrm{~cm}^{-1}$ da zeólita, a banda de absorção referente ao íon nitrato (estiramento da ligação $\mathrm{N}-\mathrm{O} 1385 \mathrm{~cm}^{-1}$ ) e deformação angular do íon amônio $\left(1400 \mathrm{~cm}^{-1}\right)$ aumentam de intensidade à medida que as quantidades ocluídas são maiores, indicando uma tendência da zeólita em agregar mais sal em seus poros, quando valores crescente são adicionados. A banda de absorção em $825 \mathrm{~cm}^{-1}$ (dobra fora do plano da ligação $\mathrm{N}-\mathrm{O}$ ), referente às deformações nos ângulos das ligações no nitrato, segue a mesma tendência durante as etapas de oclusão. ${ }^{2,15} \mathrm{Na}$ região de $2400 \mathrm{~cm}^{-1}$ surgem bandas referentes ao nitrato de amônio após a fusão do sal, o que demonstra ocorrer uma intensificação da interação do mesmo com a estrutura da zeólita, após esta etapa de oclusão. O alargamento da banda entre $3000 \mathrm{e}$ $3500 \mathrm{~cm}^{-1}$ da zeólita, nos espectros c e d, parece estar associado às interações do íon amônio (estiramento em $3142 \mathrm{~cm}^{-1}$ ) com a água contida nas cavidades da zeólita. São observadas também as bandas de deformação da ligação OH da água em $1655 \mathrm{~cm}^{-1}$, em $467 \mathrm{~cm}^{-1}$ referente às vibrações internas de deformações das ligações $\mathrm{T}-\mathrm{O}$ (anéis duplos de quatro membros, D4-R), em $557 \mathrm{~cm}^{-1}$ (vibrações externas do D4-R), em $669 \mathrm{~cm}^{-1}$ (vibrações internas de estiramento simétrico das ligações $\mathrm{T}(\mathrm{Si}, \mathrm{Al})-\mathrm{O}$ ) e em $997 \mathrm{~cm}^{-1}$ (vibrações internas de estiramento assimétrico das ligações $\mathrm{T}(\mathrm{Si}, \mathrm{Al})-\mathrm{O}){ }^{23}$

A Figura 2 apresenta as análises termogravimétricas feitas a 20 ${ }^{\circ} \mathrm{C} /$ min da zeólita ocluída com diferentes concentrações de nitrogênio (20, 40, 60, 80 e 100\% da massa de zeólita usada na oclusão). $\mathrm{Na}$ Tabela 1 estão os valores das respectivas perdas de massa a uma 
dada temperatura das amostras $20 \mathrm{~N}-4 \mathrm{~A}, 40 \mathrm{~N}-4 \mathrm{~A}, 60 \mathrm{~N}-4 \mathrm{~A}, 80 \mathrm{~N}-4 \mathrm{~A}$, $100 \mathrm{~N}-4 \mathrm{~A}$.

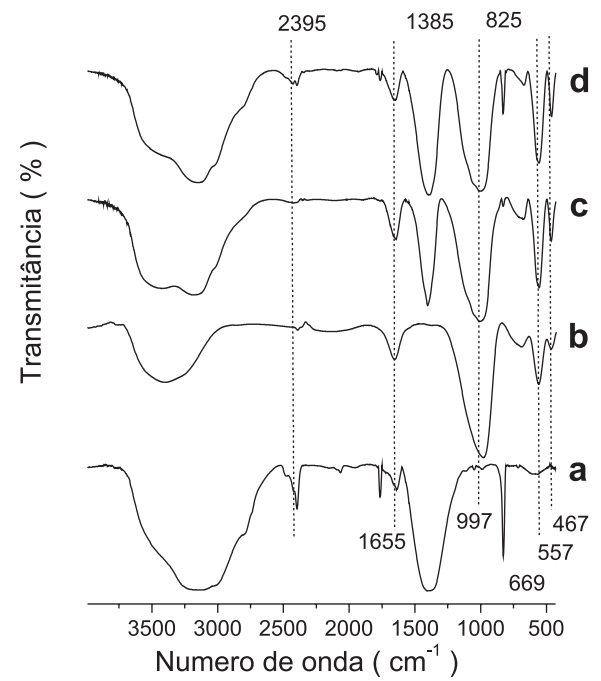

Figura 1. Espectros na região do infravermelho de amostras com e sem $\mathrm{NH}$ ${ }_{4} \mathrm{NO}_{3}$ : (a) nitrato de amônio $98 \% \mathrm{~m} / \mathrm{m}$ de pureza; (b) zeólita $\mathrm{NaA} ;($ c) após mistura física no moinho com zeólita; (d) depois da fusão do sal na zeólita

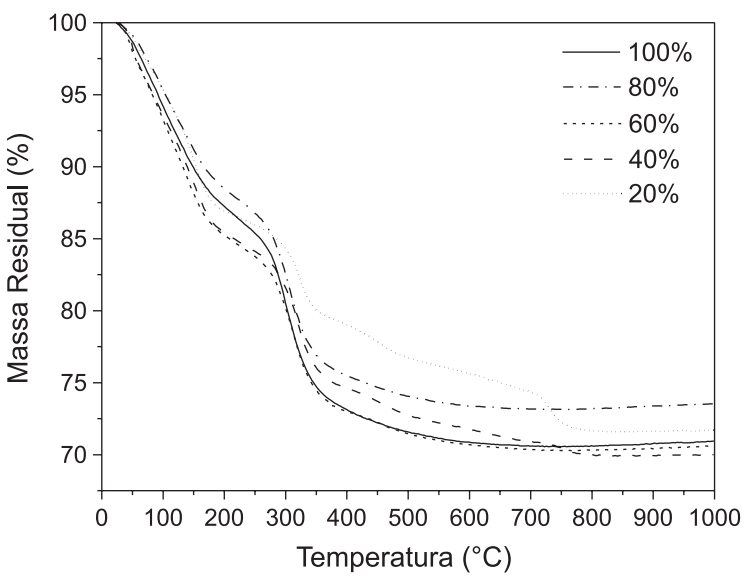

Figura 2. Análise termogravimétrica da zeólita ocluída com diferentes concentrações de nitrogênio (representadas em porcentagem da massa de zeólita usada na oclusão)
As proporções do sal ocluído na estrutura da zeólita mantiveramse como observado nos valores da perda parcial na Tabela 1 e na DTG da Figura 2, para uma temperatura de máximo em $313^{\circ} \mathrm{C}$; no entanto, a interação com água, evento que ocorre em $100^{\circ} \mathrm{C}$, é bastante variada e se deve à elevada capacidade da zeólita em absorver água.

Alguns autores têm afirmado a partir de resultados de análises térmicas (TG, DTG e DTA) do nitrato de amônio (A.R., Merck - PM $80,04 \mathrm{~g} / \mathrm{mol}$ ) que eventos que ocorrem em $170{ }^{\circ} \mathrm{C}$ estão associados à fusão do sal, e ocorre a decomposição a uma temperatura máxima de $280{ }^{\circ} \mathrm{C} .{ }^{24-27}$ Vale ressaltar que esses valores de temperatura são referentes ao sal submetido à análise térmica; no entanto, a interação deste sal com a estrutura zeolítica se apresenta distinta, devido aos diferentes tipos de poros e que podem ser acompanhados através dos deslocamentos de bandas para temperaturas mais altas. Na Tabela 1 observa-se que em concentrações menores de sal, 20 e $40 \% \mathrm{~m} / \mathrm{m}$, parece haver interações mais efetivas do nitrato de amônio com a estrutura, uma vez que ocorrem eventos tanto em $454{ }^{\circ} \mathrm{C}$, quanto em $734^{\circ} \mathrm{C}$

Na Tabela 1 evidencia-se a presença de mais eventos nas amostras com baixa concentração de nitrogênio. No entanto, devido a uma maior relação massa de nitrato ocluído/massa de zeólita, optou-se por realizar os experimentos em casa de vegetação utilizando amostras com $60 \% \mathrm{~m} / \mathrm{m}$ de $\mathrm{NH}_{4} \mathrm{NO}_{3}$ na zeólita $4 \mathrm{~A}$, uma vez que, conforme análise térmica, nesta proporção ocorreram perdas maiores em temperaturas menores, o que disponibilizaria uma quantidade mais apreciável de nitrogênio durantes os ensaios agronômicos. Outro critério é a quantidade de água liberada em $45^{\circ} \mathrm{C}$, indicando fraca adsorção de água, compatível com aquela presente somente na superfície externa da zeólita, sugerindo que o nitrato de amônio estaria ocupando todo o volume interno da zeólita.

A análise termogravimétrica da amostra $60 \mathrm{~N}-4 \mathrm{~A}$ com lavagem do sólido resultante da etapa de fusão do sal nos poros zeolíticos ( $\mathrm{N}-\mathrm{NaAs}$ s/excesso de $\mathrm{N}$ ) e sem lavagem (N-NaAc c/excesso de $\mathrm{N}$ ) pode ser observada na Figura 3 . O resultado da termogravimetria apresentado na Figura 3 mostra um perfil diferente quando comparado com o apresentado na Figura 2, devido provavelmente à quantidade de $\mathrm{NH}_{4} \mathrm{NO}_{3} / 4 \mathrm{~A}, 23$ vezes maior, permitindo melhor avaliação do que pode ocorrer com o nitrogênio.

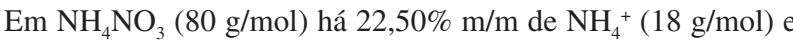
$77,50 \% \mathrm{~m} / \mathrm{m}$ de $\mathrm{NO}_{3}{ }^{-}(62 \mathrm{~g} / \mathrm{mol}) . \mathrm{O} \mathrm{NH}_{4}{ }^{+}$pode dar origem a $94 \%$ $\mathrm{m} / \mathrm{m}$ de $\mathrm{NH}_{3}(17 \mathrm{~g} / \mathrm{mol})$, segundo a reação 1 de decomposição, com estequiometria $1: 1$, ou seja, $21,25 \%$ da massa de $\mathrm{NH}_{4} \mathrm{NO}_{3}$ para $77,50 \%$ de $\mathrm{NO}_{3}^{-}$, há possibilidade de formação de $\mathrm{NO}_{2}(46 \mathrm{~g} / \mathrm{mol})$

Tabela 1. Perdas de massa observadas nos ensaios (variação da concentração de nitrogênio ocluído) e suas respectivas temperaturas máximas de decomposição

\begin{tabular}{|c|c|c|c|c|c|c|c|c|c|}
\hline \multicolumn{2}{|c|}{$20 N-4 A$} & \multicolumn{2}{|c|}{$40 N-4 A$} & \multicolumn{2}{|c|}{$60 \mathrm{~N}-4 \mathrm{~A}$} & \multicolumn{2}{|c|}{$80 \mathrm{~N}-4 \mathrm{~A}$} & \multicolumn{2}{|c|}{$100 \mathrm{~N}-4 \mathrm{~A}$} \\
\hline $\mathrm{T}\left({ }^{\circ} \mathrm{C}\right)$ & Perda $(\%)$ & $\mathrm{T}\left({ }^{\circ} \mathrm{C}\right)$ & Perda $(\%)$ & $\mathrm{T}\left({ }^{\circ} \mathrm{C}\right)$ & Perda $(\%)$ & $\mathrm{T}\left({ }^{\circ} \mathrm{C}\right)$ & Perda $(\%)$ & $\mathrm{T}\left({ }^{\circ} \mathrm{C}\right)$ & Perda $(\%)$ \\
\hline 147,00 & 13,60 & 50,10 & 3,00 & 45,34 & 3,24 & 89,60 & 12,02 & 93,50 & 13,04 \\
\hline 321,50 & 6,90 & 153,00 & 12,20 & 136,00 & 12,74 & 310,00 & 14,60 & 296,00 & 15,01 \\
\hline 452,00 & 3,50 & 321,50 & 12,73 & 316,60 & 14,10 & - & - & - & - \\
\hline 729,00 & 4,50 & 748,30 & 2,20 & - & - & - & - & - & - \\
\hline \multicolumn{2}{|c|}{ Perda Parcial $(\%) *$} & \multicolumn{2}{|c|}{ Perda Parcial $(\%) *$} & \multicolumn{2}{|c|}{ Perda Parcial $(\%) *$} & \multicolumn{2}{|c|}{ Perda Parcial $(\%) *$} & \multicolumn{2}{|c|}{ Perda Parcial $(\%) *$} \\
\hline \multicolumn{2}{|c|}{10,4} & \multicolumn{2}{|c|}{12,7} & \multicolumn{2}{|c|}{14,1} & \multicolumn{2}{|c|}{14,6} & \multicolumn{2}{|c|}{15,0} \\
\hline \multicolumn{10}{|c|}{ Rendimento de oclusão** } \\
\hline \multicolumn{2}{|c|}{62,4} & \multicolumn{2}{|c|}{44,5} & \multicolumn{2}{|c|}{37,6} & \multicolumn{2}{|c|}{32,8} & \multicolumn{2}{|c|}{30,0} \\
\hline \multicolumn{2}{|c|}{ Perda Total (\%) } & \multicolumn{2}{|c|}{ Perda Total (\%) } & \multicolumn{2}{|c|}{ Perda Total $(\%)$} & \multicolumn{2}{|c|}{ Perda Total $(\%)$} & \multicolumn{2}{|c|}{ Perda Total $(\%)$} \\
\hline \multicolumn{2}{|c|}{28,5} & \multicolumn{2}{|c|}{30,1} & \multicolumn{2}{|c|}{30,1} & \multicolumn{2}{|c|}{26,6} & \multicolumn{2}{|c|}{28,1} \\
\hline
\end{tabular}

*Não é considerado eventos que podem estar entre $40-150{ }^{\circ} \mathrm{C}$ e acima de $700{ }^{\circ} \mathrm{C}$, perda de água. $* *$ Considera somente a perda parcial, que trata dos eventos relacionados com o nitrogênio. 


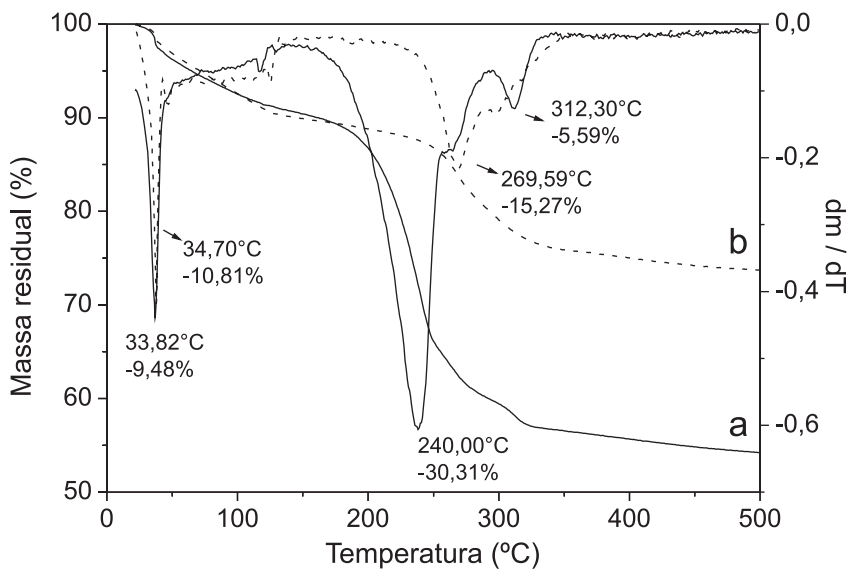

Figura 3. Análise termogravimétrica da amostra 60 -4A sem lavagem (com excesso de $N$ ) do sólido resultante da etapa de fusão do sal nos poros zeolíticos (a) e com lavagem (b)

com $74 \%$ de $\mathrm{NO}_{3}^{-}$(reação 2), ou seja, 57,50\% de $\mathrm{NH}_{4} \mathrm{NO}_{3}$. Sendo

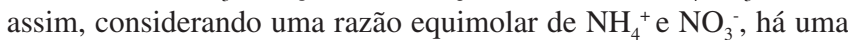
razão $\mathrm{NO}_{3}^{-} / \mathrm{NH}_{4}^{+}$de 2,70 . Desta forma, pode-se associar a uma perda total de nitrogênio da ordem de $35,9 \% \mathrm{~m} / \mathrm{m}$ e $22,6 \% \mathrm{~m} / \mathrm{m}$ referentes à formação de $\mathrm{NO}_{2}$, e $13,3 \% \mathrm{~m} / \mathrm{m}$ de $\mathrm{NH}_{3}$.

$\mathrm{NH}_{4}^{+}{ }_{(\mathrm{aq})}+\mathrm{H}_{2} \mathrm{O} \Leftrightarrow \mathrm{NH}_{3(\mathrm{~g})}+\mathrm{H}_{3} \mathrm{O}^{+}{ }_{(\mathrm{aq})}$ (reação 1)

$2 \mathrm{NO}_{3{ }^{-}(\mathrm{s})}+2 \mathrm{H}_{3} \mathrm{O}^{+} \Leftrightarrow \mathrm{NO}_{2(\mathrm{~g})}+\mathrm{O}_{2(\mathrm{~g})}+\mathrm{NO}_{(\mathrm{g})}+3 \mathrm{H}_{2} \mathrm{O} \quad$ (reação 2)

O estudo cinético baseado no modelo de Osawa teve como princípio o processo termodegradativo da amostra $60 \% \mathrm{~N}-\mathrm{NaA}$ em cinco razões de aquecimento distintas. Para 76, 78, 80, 82, 84, 86, 88, 90, 92, 94 e $96 \%$ de massa percentual, foi obtido um gráfico $\log \beta$ versus $1 / \mathrm{T}\left(\mathrm{K}^{-1}\right)$, a partir do qual foram determinados os parâmetros cinéticos.

Para as curvas TG's da amostra 60N-4A em diferentes razões de aquecimento, obtidas em atmosfera de ar sintético $\left(80 \% \mathrm{~N}_{2}\right.$ e $20 \%$ $\mathrm{O}_{2} ; 5.0 \mathrm{FID}$ ), se aproximando das condições de cultivo (presença de oxigênio), há formação de nitrito a partir do íon amônio e, consequentemente, nitrato pela reação do nitrito com oxigênio. $\mathrm{O}$ nitrato formado reage em meio ácido sendo reduzido a nitrogênio molecular ou a íons amônio. A extensão da decomposição é maior em razões da baixa razão de aquecimento, já que nestas condições, o calor é melhor absorvido pela estrutura.

Os resultados do estudo cinético da amostra $40 \% \mathrm{~N}-\mathrm{NaA}$ foram realizados em atmosfera de ar sintético (80\% $\mathrm{N}_{2}$ e $20 \% \mathrm{O}_{2} ; 5.0$ FID). Baseado no método proposto por Osawa, o coeficiente angular (B) de cada reta $(\log \beta$ versus $1 / \mathrm{T})$ é representado pela equação: $\mathrm{B}=$ $\Delta \log \beta / \Delta(1 / \mathrm{T})$. A razão $\mathrm{R} / \mathrm{b}$ (ou 8,314/0,457) é pré-estabelecida como sendo uma "constante" aproximadamente igual a 18,2 J/mol. Logo, pela Equação 2, pode-se calcular a energia de ativação para cada perda percentual de massa (Figura 4).

Pela Equação 1, pode-se calcular a energia de ativação para cada perda percentual de massa. A Figura 4 apresenta um gráfico da energia de ativação versus massa residual das amostras $40 \% \mathrm{~N}-\mathrm{NaA}$ e $60 \% \mathrm{~N}$ $\mathrm{NaA}$, e fornece informações precisas quanto às energias envolvidas nas perdas de massa e respectivas temperaturas.

As curvas da Figura 4 evidenciam que todas as substâncias que não fazem parte da estrutura da zeólita são eliminadas com o emprego de diferentes quantidades de energia e se observam patamares desta energia, indicando a existência de substâncias cuja forma de interação com a estrutura do material é contínua e que o material está associado homogeneamente à estrutura da zeólita. O primeiro patamar de energia foi atribuído à eliminação de água, observando-se que não

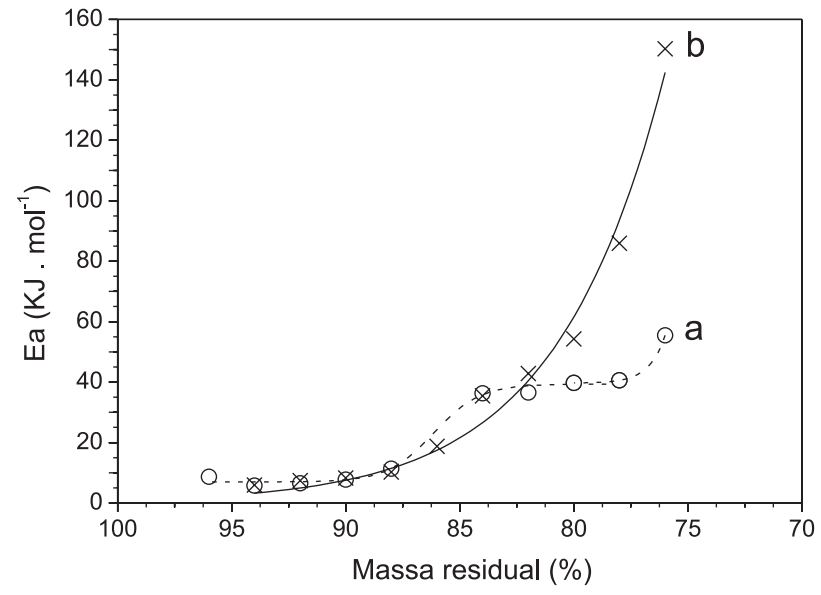

Figura 4. Massa versus energia de ativação para os processos termodegradativos da amostra 40N-4A (-×-) e 60N-4A (--O--)

há variação expressiva na energia de ativação, esta é em média 7,5 $\mathrm{KJ} \mathrm{mol}^{-1}$, ou seja, a água deve estar fracamente associada à estrutura da zeólita. O segundo patamar de energia foi associado à amônia e dióxido de nitrogênio com valores de energia de ativação em torno de $40 \mathrm{KJ} \mathrm{mol}^{-1}$, estando mais fortemente ligado à estrutura zeólitica; sua perda se dá até aproximadamente $500{ }^{\circ} \mathrm{C}$, sendo que a partir daí ocorre estabilização das curvas TG's. ${ }^{28}$

Os resultados cinéticos da amostra 40N-4A indicaram interações mais fortes que as observados na amostra 60N-4A, apesar da mesma apresentar valores maiores de perda de massa parcial e total. Os valores médios de energia de ativação para a amostra $60 \mathrm{~N}-4 \mathrm{~A}$ ficaram em torno de $10 \mathrm{KJ} \mathrm{mol}^{-1}$ nos primeiros momentos de perdas de massa, relativamente próximo do observado na Figura 4 (7,5 KJ $\left.\mathrm{mol}^{-1}\right)$, também associado à perda de água, no entanto, perda de massa acima de $12,5 \% \mathrm{~m} / \mathrm{m}$, a energia de ativação é variável e atribuída a transformações, fusões e decomposição do sal ocorrendo em faixas de temperaturas similares à da amostra $60 \mathrm{~N}-4 \mathrm{~A}$ com sobreposição de eventos.

Devido à porosidade regular e dimensões definidas apresentadas pela estrutura da zeólita 4A, a oclusão do nitrato de amônio depende num primeiro momento das dimensões, tanto dos íons, quanto do diâmetro das janelas dos poros, pois deverá haver um livre acesso dos íons para o interior dos poros da zeólita. ${ }^{29}$

Como as características estruturais desta zeólita favorecem a oclusão de uma maior quantidade de sal, entende-se que a zeólita 4A proporciona resultados próximos dos observados por Park e colaboradores. ${ }^{15}$

A liberação de amônia com a retenção de $\mathrm{H}^{+}$(reação 1) pela estrutura da zeólita tem sido considerada como um fenômeno muito importante, pois evitaria o aumento da acidez do solo. Acredita-se que a decomposição do nitrato em óxido ocorra devido à polarização do íon nitrato pelos cátions intrazeolíticos. O poder polarizante dos cátions neste caso é muito importante, já que $\mathrm{NO}_{3}$ - é uma base dura e, portanto, não é facilmente polarizável. Quanto mais polarizante o cátion, mais fácil será a decomposição do nitrato.

Os resultados referentes à caracterização do solo são apresentados a seguir.

O solo foi classificado como franco argilo arenoso, de acordo com a classificação textural dos solos, com os seguintes teores de areia, silte e argila: 535,6; 152,1 e 312,3 $\mathrm{g} \mathrm{kg}^{-1}$, respectivamente. ${ }^{21}$

$\mathrm{O}$ pH e a condutividade em suspensão aquosa foram, respectivamente, 7,73 e $0,2 \mathrm{mS} / \mathrm{cm}$, em KCl 6,92 e 115,6 mS/cm e em $\mathrm{CaCl}_{2}$ 7,15 e $2468,0 \mathrm{mS} / \mathrm{cm}$

A análise termogravimétrica (TG e DTG) do solo está repre- 
sentada na Figura 5. A interação entre o $\mathrm{NH}_{4} \mathrm{NO}_{3}$ e a zeólita ocorre em regiões distintas da estrutura porosa (janelas, grades, canais, cavidades, largura de poro) e de forma lenta. ${ }^{30}$

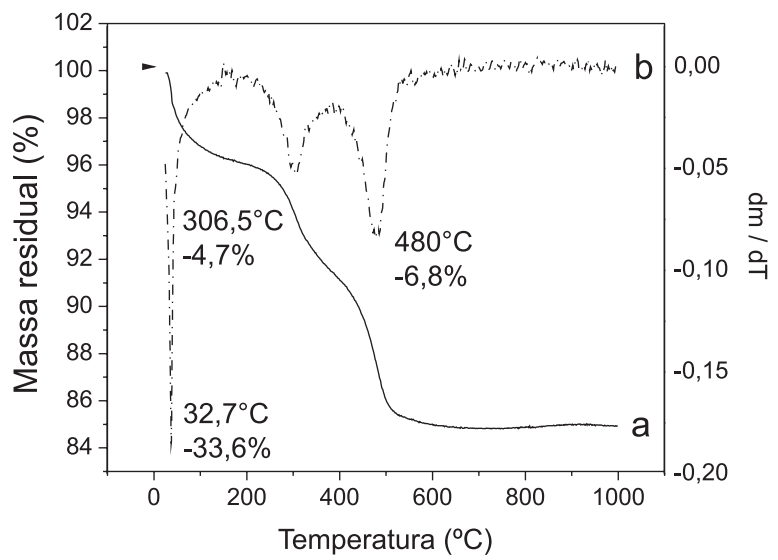

Figura 5. Termogravimetria do solo (a) e derivada da termogravimetria do solo (b)

A análise termogravimétrica do solo apresentou três eventos envolvendo perdas de massa, o primeiro em $32,7^{\circ} \mathrm{C} \operatorname{com} 3,6 \% \mathrm{~m} / \mathrm{m}$ de perda, o segundo em $306,5^{\circ} \mathrm{C} \operatorname{com} 4,7 \% \mathrm{~m} / \mathrm{m}$ e o terceiro em 480 ${ }^{\circ} \mathrm{C}$ com 6,8\% m/m (Figura 5). A perda de massa total de $15,1 \% \mathrm{~m} / \mathrm{m}$ foi atribuída à perda ao fogo: água adsorvida, substâncias húmicas e hidroxila estrutural.

Os resultados de fluorescência de raios- $\mathrm{X}$ do solo apresentaram a seguinte composição em $\mathrm{g}$ por $\mathrm{dm}^{3}: 269,7 \mathrm{Si} ; 258,9 \mathrm{Fe} ; 92,2 \mathrm{Mg}$; 274,6 K; 131,1 Al; 156,5 Ti; 116,8 Ca; 0,6 Ni; 2,1 P; 0,9 S; 4,2 Mn.

$\mathrm{O}$ difratograma de raios- $\mathrm{X}$ do solo pode ser observado na Figura 6. Analisando os resultados de fluorescência e de difratograma de raios-X do solo (Figura 6) pode-se avaliar a amostra com prévia identificação das fases cristalográficas (software X'Pert HighScore) como sendo solo predominantemente caulinítico, picos coincidentes com o padrão cristalográfico da literatura. ${ }^{31}$ Devido o solo ser de origem calcária, e juntamente com o alto teor de ferro observado na fluorescência, verifica-se também a presença de goethita associada à amostra. Tal composição está coerente com a razão Si:Al de 1,97 e com os valores médios de $\mathrm{pH}$ e condutividade obtidos em triplicata, com o solo se apresentando levemente alcalino.

A germinação ocorreu do $4^{\circ}$ ao $5^{\circ}$ dia após o plantio. Todos os tratamentos apresentaram em média $4,7 \pm 0,5$ sementes germinadas.

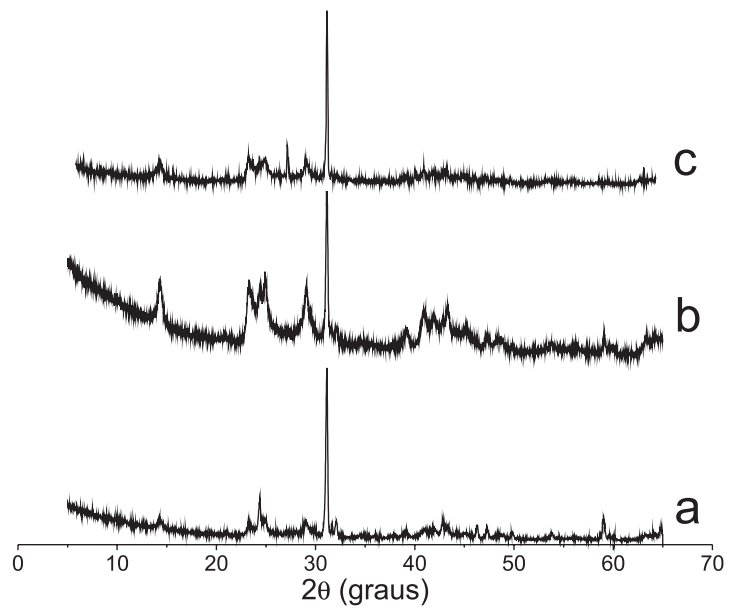

Figura 6. Difratograma de raios-X do solo fração argila (a), fração silte (b) e in natura (c)
A seguir são apresentadas imagens fotográficas (Figura 7), em mesma escala, do resultado final do cultivo para cada fonte de nitrogênio (mesma quantidade) utilizada. As características visuais dos tratamentos apresentados na Figura 7 não permitiram diferenciações significativas em relação à altura e ao diâmetro do colmo; no entanto, os tratamentos na presença de zeólita ocluída com nitrogênio apresentaram folhas apicais mais largas com verde mais intenso, sendo um indicativo de maior teor de nitrogênio em relação ao tratamento com fertilizante comercial $\left(\mathrm{NH}_{4} \mathrm{NO}_{3}\right)$.

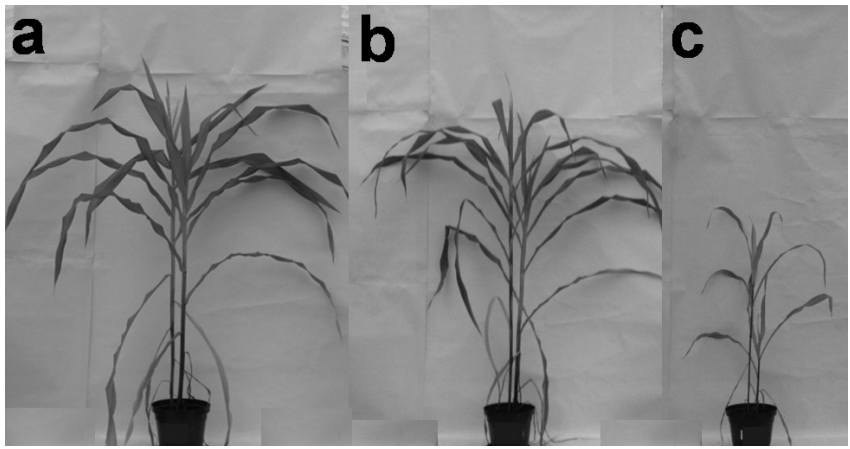

Figura 7. Foto das plantas cultivadas utilizando diferentes tratamentos: cultivo com zeólita ocluída com nitrogênio em excesso (a); cultivo com fertilizante comercial, $\mathrm{NH}_{4} \mathrm{NO}_{3}(b)$ e a testemunha, sem nitrogênio (c)

Os resultados estatísticos para avaliar a produção de matéria seca apresentaram diferenças entre um ou dois tratamento, mas que se relacionam em igualdade estatística com os demais valores. No $1^{\circ}$ desbaste não foi possível avaliar com precisão as diferenças, pois a quantidade absorvida de nitrogênio nesta fase da cultura ainda é pequena. No entanto, fica claro que a produção de matéria seca do $2^{\circ}$ desbaste não sofreu influência da concentração inicial de nitrogênio, pois apesar do IPR ter sido significativamente maior no tratamento com $\mathrm{NH}_{4} \mathrm{NO}_{3}$ no $1^{\circ}$ desbaste, os valores de IPR para o $2^{\circ}$ desbaste não variaram de forma significativa.

A produção de matéria seca do $2^{\circ}$ desbaste em diferentes concentrações de nitrogênio se mostrou constante, não variando significativamente no tratamento com nitrato de amônio ocluído. Diferentemente, o tratamento na ausência de zeólita apresenta variações mais altas entre as produções, uma vez que a produção de MS está ligada ao acúmulo de nitrogênio. ${ }^{32}$

Consideram-se dois aspectos para aplicação de uma fonte de nitrogênio eficiente, aproveitamento e perda por lixiviação; já que menos de $50 \%$ é utilizado pelas culturas, o percentual de aproveitamento foi calculado com base no teor de nitrogênio total na matéria seca, visto que as plantas absorvem predominantemente nitrogênio oriundo do solo. ${ }^{33}$

As médias da produção de matéria seca para diferentes fertilizantes nitrogenados e quantidade de nitrogênio aplicado no solo, presente na parte aérea e lixiviado de cada tratamento, são apresentadas nas Tabelas 2 e 3, respectivamente.

Os resultados do método estatístico na análise de variância para a eficiência no aproveitamento de nitrogênio empregado indicaram não haver diferença entre os tratamentos (média geral igual a 68,4\% de aproveitamento) a 5\% de probabilidade, com CV (coeficiente de variância) de $18,63 \%$ e DMS (diferença mínima significativa) igual a $29,3 \%$. O teste $\mathrm{F}$ apresentou-se significativo para $14,25 \%$ de probabilidade. Há uma probabilidade superior a $85,75 \%$ de que pelo menos um contraste entre médias de tratamento difira de zero. Isto significa que os efeitos de tratamento somente serão estatisticamente diferentes, havendo resposta para tratamentos, em nível de significância maior que $14,25 \%$. 
Tabela 2. Médias ${ }^{1}$ da produção de matéria seca (MS) para diferentes fertilizantes nitrogenados (38 dias de cultivo)

\begin{tabular}{|c|c|c|c|c|c|c|}
\hline \multicolumn{7}{|c|}{ Fertilizante } \\
\hline \multicolumn{3}{|c|}{ N-NaA com excedente de N (g) (massa de nitrogênio em mg) } & \multicolumn{3}{|c|}{$\mathrm{NH}_{4} \mathrm{NO}_{3}$} & \multirow{2}{*}{$\begin{array}{c}\text { Testemunha } \\
0,00\end{array}$} \\
\hline $5,57(700)$ & 4,07 (511) & $2,57(300)$ & $2,00(700)$ & $1,46(511)$ & $0,92(300)$ & \\
\hline \multicolumn{7}{|c|}{$\mathrm{MS} \pm \mathrm{SD}^{2}\left(\mathrm{IPR}^{3}\right)$ do desbaste com 15 dias de cultivo e com 38 dias } \\
\hline $\begin{array}{c}2,53 \pm 0,71 \\
(51,09) \mathrm{ac}\end{array}$ & $\begin{array}{c}3,76 \pm 0,95 \\
(67,11) \mathrm{d}\end{array}$ & $\begin{array}{c}2,05 \pm 1,29 \\
(39,70) \mathrm{ae}\end{array}$ & $\begin{array}{c}3,25 \pm 1,04 \\
(61,90) \mathrm{cd}\end{array}$ & $\begin{array}{c}3,23 \pm 1,07 \\
(61,72) \mathrm{cd}\end{array}$ & $\begin{array}{l}2,78 \pm 0,58 \\
(55,45) \text { acd }\end{array}$ & $1,24 \pm 0,48 \mathrm{e}$ \\
\hline $\begin{array}{c}29,91 \pm 3,67 \\
(87,46) \mathrm{ab}\end{array}$ & $\begin{array}{c}29,49 \pm 0,70 \\
(87,29) \mathrm{ab}\end{array}$ & $\begin{array}{c}26,25 \pm 0,72 \\
(85,72) b\end{array}$ & $\begin{array}{c}33,68 \pm 1,22 \\
(88,87) \mathrm{a}\end{array}$ & $\begin{array}{c}30,77 \pm 2,27 \\
(87,81) a b\end{array}$ & $\begin{array}{c}24,43 \pm 5,64 \\
(84,66) b\end{array}$ & $3,75 \pm 0,79$ \\
\hline
\end{tabular}

(1) Médias seguidas da mesma letra não diferem entre si pelo teste de Tukey $(\mathrm{P}>0,05) .{ }^{(2)} \mathrm{SD}$ - desvio padrão relativo à análise em quadruplicata. ${ }^{(3)}$ IPR - incremento da produção relativa $=100-[(\mathrm{MS}$ sem N x 100)/MS com N].

Tabela 3. Quantidade de nitrogênio* aplicado no solo, presente na parte aérea e lixiviado de cada tratamento (38 dias de cultivo)

\begin{tabular}{|c|c|c|c|c|c|c|c|}
\hline Variável / Tratamentos & 1 & 2 & 3 & 4 & 5 & 6 & 7 \\
\hline Nitrogênio total aplicado no solo & 280,0 & 204,6 & 129,2 & 280,0 & 204,6 & 129,2 & 0,0 \\
\hline Nitrogênio total lixiviado & 14,8 & 3,4 & 5,6 & 19,1 & 10,4 & 7,5 & 3,5 \\
\hline Porcentagem lixiviado & 2,6 & 1,4 & 2,8 & 3,2 & 2,7 & 3,4 & - \\
\hline Nitrogênio total na MS & 15,6 & 11,2 & 9,8 & 16,7 & 10,5 & 8,9 & 1,53 \\
\hline Percentual de aproveitamento & 66,7 & 64,3 & 79,2 & 80,5 & 63,3 & 67,6 & - \\
\hline
\end{tabular}

* As quantidades de nitrogênio apresentadas para cada tratamento são abatidas da contribuição de nitrogênio proveniente do solo.

Quanto às perdas por lixiviação, os tratamentos com zeólita ocluída com $\mathrm{NH}_{4} \mathrm{NO}_{3}$ resultaram nas menores perdas de nitrogênio, como pode ser observado na Tabela 3 , sendo mais um indicativo da validação do método de cultivo para menores concentrações de nitrogênio ocluído na zeólita. ${ }^{34,35}$

Embora os tratamentos não difiram estatisticamente entre si, as menores concentrações de nitrogênio são mais apropriadas para o cultivo, visando não haver desperdício de nitrogênio. Com o mesmo objetivo, o uso de zeólita como suporte para o nitrogênio é mais apropriado devido haver menor perda por lixiviação.

\section{CONCLUSÃO}

O tratamento térmico para preparação da zeólita ocluída conduz decisivamente à mistura na qual a zeólita fica com sua estrutura saturada pelo nitrato de amônio. Este sal interage com os vários poros da zeólita, agregando-se de modo distinto, de forma que esta característica irá estabelecer domínio sobre a propriedade de liberação da estrutura, com o emprego de diferentes quantidades de energia, tornando-a controlável e lenta ao longo do tempo, para uso na agricultura.

As características físicas e químicas do solo favorecem seu uso como suporte para a zeólita ocluída com $\mathrm{NH}_{4} \mathrm{NO}_{3}$, apresentando predominantemente na sua composição caulinita em meio próximo da neutralidade, porém levemente alcalina, e atividade iônica correspondente à geoquímica do solo. Desta forma, pode haver controle do sistema, a partir da zeólita como substrato dominante para a liberação lenta e controlada, suprindo as necessidades nutricionais de nitrogênio, sem perdas apreciáveis de nitrogênio.

A análise estatística mostrou que não há diferença entre os tratamentos a 5\% de probabilidade. O tratamento envolvendo zeólita ocluída com nitrogênio no cultivo de milho demonstrou haver melhor aproveitamento de nitrogênio em relação ao índice de produção relativa e pequenas perdas por lixiviação, não havendo necessidade de utilizar altos teores de nitrogênio no cultivo.

\section{AGRADECIMENTOS}

À CAPES e ao CNPq pelo suporte financeiro.

\section{REFERÊNCIAS}

1. Rehakova, M.; Cuvanova, S.; Dzivak, M.; Rimar, J.; Gaval'ova, Z.; Curr. Opin. Solid State Mater. Sci. 2004, 8, 397.

2. Park, M.; Kim, J. S.; Choi, C. L.; Kim, J. E.; Heo, N. H.; Komarneni, S.; Choi, J.; J. Controlled Release 2005, 106, 44.

3. Larsen, S. C.; J. Phys. Chem. C 2007, 111, 18464.

4. Macias, A. F.; Spinola, A. G.; Mendoza, T. M. H.; Gonzalez, F. D.; Zelaya, F. P.; Interciencia 2007, 32, 692.

5. Bansiwal, A. K.; Rayalu, S. S.; Labhasetwar, N. K.; Juwarkar, A. A.; Devotta, S.; J. Agric. Food Chem. 2006, 54, 4773.

6. Carvalho, A.; Pires, J.; Veloso, P.; Machado, M.; Carvalho, M. B.; Rocha, J.; Microporous Mesoporous Mater. 2003, 58, 163.

7. Park, M.; Choi, C. L.; Kim, J. S.; Lee, D. H.; Kim, K. S.; Heo, N. H.; Choi, J.; Microporous Mesoporous Mater. 2003, 62, 1.

8. Komarneni, S.; Newalkar, B. L.; Li, D. S.; Gheyi, T.; Lopano, C. L.; Heaney, P. J.; Post, J. E.; J. Porous Mater. 2003, 10, 243.

9. Zendehdel, M.; Kooti, M.; Amini, M. M.; J. Porous Mater. 2005, 12, 143.

10. Kubota, A.; Hoshiba, K.; Bordon, J.; Rev. Bras. Ciênc. Solo 2008, 32, 1627. 
11. Bernardi, A. C. D. C.; Werneck, C. G.; Haim, P. G.; Botrel, N.; OianoNeto, J.; Monte, M. B. D. M.; Verruma-Bernardi, M. R.; Hortic. Bras. 2007, 25, 306.

12. Bernardi, A. C. D. C.; Werneck, C. G.; Haim, P. G.; Rezende, N. D. G. D. A. D. M.; Paiva, P. R. P.; Monte, M. B. D. M.; Rev. Bras. Frutic. 2008, 30, 794.

13. Mohammad, M. J.; Karam, N. S.; Al-lataifeh, N. K.; Commun. Soil Sci. Plant Anal. 2004, 35, 2283.

14. Bernardi, A. C. C.; Bernardi, M. R. V.; Werneck, C. G.; Haim, P. G.; Monte, M. B. M.; Hortic. Bras. 2005, 23, 920.

15. Park, M.; Shin, S. C.; Choi, C. L.; Lee, D. H.; Lim, W. T.; Komarneni, S.; Kim, M. C.; Choi, J.; Heo, N. H.; Microporous Mesoporous Mater. 2001, 50, 91.

16. Andrade, J. C. R. de A.; Romero, R. E.; Soares, I.; Silva, L. R. D. da; Quim. Nova 2010, 33, 1692.

17. Mothé, C. G.; Azevedo, A. D.; Análise Térmica de Materiais, $1^{\mathrm{a}}$ ed., Ieditora: São Paulo, 2002

18. Amaro Filho, J.; Assis Júnior, R. N.; Mota, J. C. A.; Física do Solo: Conceitos e Aplicações, Imprensa Universitária - Escola de Agronomia do Ceará: Fortaleza, 2008.

19. Carolino, J. T.; Dissertação de Mestrado, Universidade Federal do Ceará, Brasil, 1995

20. Silva, L. I. D. D.; Carneiro, M. C.; Emídio, V. D. S.; Henrique Jr., S. D. S.; Monteiro, M. I. C.; Quim. Nova 2006, 29, 46.

21. Rolim, G. D. S.; Camargo, M. B. P. D.; Lania, D. G.; Moraes, J. F. L. D.; Bragantia 2007, 66, 711 .
22. Yamada, H.; Yokoyama, S.; Watanabe, Y.; Uno, H.; Tamura, K.; Sci. Technol. Adv. Mater. 2005, 6, 394.

23. Huang, Y. N.; Jiang, Z. M.; Microporous Mater. 1997, 12, 341.

24. Rubtsov, Y. I.; Kazakov, A. I.; Kirpichev, E. P.; Lempert, D. B.; Manelis, G. B.; Russ. J. Appl. Chem. 2005, 78, 870.

25. Shen, L. J.; Wang, X. G.; J. University Sci. Technol. Beijing 2005, 12, 12.

26. Lang, A. J.; Vyazovkin, S.; Mater. Lett. 2008, 62, 1757.

27. Ryshchenko, I. M.; Kulatskii, N. S.; Savenkov, A. S.; Protiven, I. N.; Ratushnaya, L. N.; Russ. J. Appl. Chem. 2008, 81, 743.

28. Chandrasekhar, S.; Pramada, P. N.; Ceram. Int. 2001, 27, 351.

29. Kithome, M.; Paul, J. W.; Lavkulich, L. M.; Bomke, A. A.; Soil Sci. Soc. Am. J. 1998, 62, 622.

30. Esposito, S.; Ferone, C.; Pansini, M.; Bonaccorsi, L.; Proverbio, E.; J. Eur. Ceram. Soc. 2004, 24, 2689.

31. Lounis, Z.; Djafri, F.; Bengueddach, A. E. K.; Annales de ChimieScience des Materiaux 2006, 31, 439.

32. Binford, G. D.; Blackmer, A. M.; Meese, B. G.; Agronomy J. 1992, 84, 881.

33. Bredemeier, C.; Mundstock, C. M.; Cienc. Rural 2000, 30, 365.

34. Beltrão, N. E. D. M.; Fideles Filho, J.; Figueirêdo, I. C. D. M.; Rev. Bras. Eng. Agric. Ambient. 2002, 6, 547.

35. Ahmed, O. H.; Husin, A.; Hanif, A. H. M.; Acta Agriculturae Scandinavica Section B-Soil and Plant Science 2008, 58, 182. 